\title{
Rib 9
}

National Cancer Institute

\section{Source}

National Cancer Institute. Rib 9. NCI Thesaurus. Code C52759.

The ninth rib counting from the top of the rib cage down. 